Revista de Psicología y Educación / Journal of Psychology and Education, 2022, 17(1), 69-78 (www.rpye.es) Doi: https://doi.org/10.23923/rpye2022.01.217

ISSN: $1699-9517$

\title{
Factores psicológicos y consecuencias del Síndrome Fear of Missing Out: Una Revisión Sistemática
}

\author{
José Gabriel Soriano-Sánchez
}

Universidad de La Rioja

\begin{abstract}
Resumen: En los últimos años, el uso excesivo de teléfonos inteligentes se ha convertido en un importante desafío para la salud pública. En esta dirección, ha surgido el Síndrome llamado FoMO, por su expresión en inglés Fear of Missing Out (FoMO), originándose por la aparición de angustia a consecuencia de una falta de experiencias gratificantes. De este modo, surge el objetivo del presente trabajo, que consiste en identificar mediante una revisión sistemática los factores psicológicos asociados a este síndromeF. Para alcanzarlo, se han utilizado diferentes bases de datos de carácter internacional, siendo las bases elegidas Web of Science, Scopus y PsycINFO. Tras aplicar los criterios de inclusión y exclusión establecidos, quedaron 11 investigaciones incluidas para revisión sistemática. Los resultados de los diferentes trabajos revelan que el uso problemático de Internet parece mediar entre en la presencia del síndrome. En conclusión, la presencia del Síndrome FoMO media sobre la salud mental de la persona. Particularmente, en la aparición de sentimientos de soledad, ansiedad, depresión, bienestar emocional, calidad del sueño y consumo de alcohol. Por tanto, se sugiere que se sigan realizando nuevos trabajos, de manera que brinden la oportunidad de abordar este problema actual.
\end{abstract}

Palabras clave: FoMO; factores psicológicos; predictores; revisión sistemática; Internet.

\section{Psychological Factors and Consequences of Fear of Missing Out Syndrome: A Systematic Review}

Abstract: In recent years, the excessive use of smartphones has become a major public health challenge. In this context, the syndrome called the Fear of Missing Out (FoMO) has arisen, characterized by the emergence of anxiety as a result of a lack of rewarding experiences. The aim of the present work is to identify through a systematic review the psychological factors associated with the fear of missing an event. For this purpose, the Web of Science, Scopus, and PsycINFO international databases were used. After applying the established inclusion and exclusion criteria, 11 investigations were included for the systematic review. The results of the different studies reveal that the problematic use of the Internet seems to mediate the presence of the syndrome. In conclusion, the presence of the FoMO syndrome mediates in a person's mental health, particularly, in the emergence of feelings of loneliness, anxiety, depression, lack of emotional well-being, quality of sleep, and alcohol consumption. Therefore, it is suggested that further work be continued to address this problem.

Key words: FoMO; psychological factors; predictors; systematic review; Internet.

En los últimos años, el uso excesivo de teléfonos inteligentes se ha convertido en importantes desafíos para la salud pública, dada su relación con problemas mentales (Elhai et al., 2016). En esta dirección, ha

Recibido: 10/11/2021 - Aceptado: 11/01/2022 - Avance online:20/01/2022 *Correspondencia: José Gabriel Soriano Sanchez

Universidad de la Rioja Dirección: 23630, (Villargordo) (Jaén), España. E-mail: josoris@unirioja.es

Soriano-Sánchez, J. G. (2022).Factores psicológicos y consecuencias del Síndrome Fear of Missing: Una Revisión Sistemática. Revista de Psicología y Educación, 17(1), 69-78, https:/4 doi.org/10.23923/rpye2022.01.217 surgido el síndrome llamado FoMO, por su expresión en inglés Fear of Missing Out que traducido al castellano significa "miedo a perderse algún acontecimiento", originándose por la aparición de angustia a consecuencia de una falta de experiencias gratificantes. Otros autores definen el Síndrome FoMO como una práctica amplificada de la adicción a Internet (Baker et al., 2016; Przybylsky et 
al., 2016). Al usuario le surge la necesidad de mantenerse conectado al uso de las Tecnologías de la Información y Comunicación (TIC) y Redes Sociales (RRSS), para observar cómo lo están pasando el resto de familiares, amigos y/o conocidos, mediante sus publicaciones en los diferentes perfiles virtuales como, por ejemplo, Facebook, Twitter e Instagram (Budnick et al., 2020; Elhai et al., 2020). Y, es que, tal y como revelan Brito et al. (2021) que, entre las distintas ventajas de la utilización de las TIC y uso de Internet, se encuentra una nueva forma de comunicación ubicua e instantánea, con cualquier persona y lugar.Particularmente, la etiología del FoMO puede deberse a una falta de cercanía con los demás. Es decir, de relaciones interpersonales presenciales, lo que llega a causar una serie de trastornos de ansiedad relacionados con lo que otros hacen mientras el que visualiza la RRSSse encuentra fuera de la experiencia (Coiro et al., 2017). En este sentido, trabajos como el realizado por Ferreira et al. (2021), ha indicado que las personas que muestran una peor utilización del uso de teléfonos inteligentes e Internet, presentan un peor ajuste emocional y mayores niveles de ansiedad. Además, este síndrome se ha visto relacionado con una serie de resultados negativos que incluyen la insatisfacción con la vida, el mal humor y el sueño desregulado (Riordan et al., 2015). Este hecho ha dado lugar a que en el último año emerian nuevas investigaciones en el campo de la educación, puesto que la salud del estudiante puede verse afectada, influyendo de manera negativa en sus relaciones sociales y en su estado psicológico y físico (Adams et al., 2020; Dhir et al., 2018; Tang et al., 2020).

En los primeros años de la adolescencia los padres realizan un mayor control o vigilancia sobre los hijos e hijas en relación al uso de teléfonos móviles e Internet. Sin embargo, con el paso del tiempo se va perdiendo, lo que conlleva a que los adolescentes puedan experimentar relaciones sociales de riesgo con desconocidos a través de la red (Barry et al., 2017). Ello puede posibilitar que se incremente su adicción al uso de RRSS (Montes-Vozmediano et al., 2020). A su vez, la literatura previa ha revelado que las personas que se encuentran más preocupadas por mantener una imagen positiva en las RRSS muestras una mayor discrepancia percibida entre su identidad online y fuera de línea (Robinson et al., 2019; Scalzo y Martinez, 2017). Por otro lado, se ha descubierto que usuario de Internet con un mayor uso de RRSS presentan un mayor aislamiento social, independientemente de la edad (Primack et al., 2017). Al parecer la presencia de Síndrome FoMO conlleva a una utilización excesiva de Apps, tanto en población joven como adulta (Barry et al., 2019; Reer et al., 2019). No obstante, no cabe duda de que los dispositivos inteligentes como móviles, Tablets $u$ ordenadores, ofrecen importantes beneficios para los usuarios, como podrían ser sobre la comunicación social (Chan, 2015), la mejora de la calidad educativa (Godwin-Jones, 2011) $\mathrm{O}$, incluso, en la asistencia sanitaria (Camacho et al., 2014).

Además, el uso problemático o abusivo de las TIC e Internet puede tener consecuencias negativas en el ámbito académico, como distracción en el aprendizaje y bajo rendimiento académico (Rozgonjuk et al., 2018). Así como, puede generar una disminución de la productividad en la esfera profesional (Duke y Montag, 2017). Por tanto, sería interesante que las personas de la era digital recibiesen información y estrategias no solamente sobre los beneficios que conllevan las $\mathrm{TIC}$, sino también en la formación de estrategias de uso responsable, tanto en la familia, como en la escuela y la sociedad (Martínez, 2021), para realizar un uso adecuado y evitar la aparición del síndrome (Tang et al., 2020).

A partir de lo anterior, surge nuestro principal objetivo en el presente trabajo que consiste en identificar mediante una revisión sistemática los factores psicológicos predictores del síndrome "Fear Of Missing Out" (FoMO). Y, concretamente, como objetivos específicos: a) identificar variables predictores del Síndrome FoMO; b) analizar consecuencias del Síndrome FoMo; y c) presentar resultados de evidencia científica que permitan intervenir sobre este problema de salud pública. 


\section{METODOLOGÍA}

\section{PROCEDIMIENTO Y ESTRATEGIAS BÚSQUEDA}

Para la realización de la presente revisión sistemática se han seguido los pasos propuestos por la declaración PRISMA (Moher et al., 2009). Para ello, se realizó una búsqueda de investigaciones en diferentes recursos electrónicos de carácter científico. Concretamente, las bases de datos utilizadas fueron Web of Science, Scopus y PsycINFO, donde se empleó la siguiente fórmula de búsqueda en inglés: (Fear of Missing Out) AND (health). Asimismo, de manera general, en los tres recursos electrónicos utilizados se empleó el filtro "artículo" y, de manera específica, en Scopus se aplicó el filtro "psicología" dentro del apartado área temática y en PsycINFO "estudio empírico".

Respecto a la limitación temporal, no se empleó ningún filtro, con el fin de evitar riesgo de sesgo en relación al tiempo de publicación. No obstante, el primer trabajo publicado en relación con los criterios de publicación establecidos tuvo lugar en 2016, siendo realizadas las últimas publicaciones en el año 2020. Ello sugiere a un creciente interés respecto a la temática planteada en el presente trabajo.

En último lugar, se procedió a la lectura de cada uno de los títulos y resúmenes. No obstante, en los casos donde hubo alguna duda, se pasó a la lectura completa del trabajo. Por otro lado, en relación a la fecha de búsqueda, se efectuó el 22 de junio de 2020.

Para facilitar la incorporación de referencias y bibliografía al presenta trabajo, se hizo uso de la herramienta informática Mendeley, propuesta por Elsevier (2019).

\section{CRITERIOS DE INCLUSIÓN Y EXCLUSIÓN}

Teniendo en cuenta el objetivo general del presente trabajo, se establecieron los siguientes criterios:
Se incluyeron los trabajos que cumplían los siguientes criterios: (a) trabajos publicados en inglés y español; (b) estudios de evidencia empírica; (c) trabajos que evalúen la predicción entre FoMO y diferentes parámetros psicológicos como depresión, ansiedad, etc.; y (d) que empleen análisis estadísticos de regresión.

Los criterios de exclusión establecidos fueron: (a) trabajos teóricos; (b) investigaciones publicadas en un idioma que no sea inglés o español; (c) trabajos relacionados con otros asuntos relacionados con salud (de enfermedades), uso de internet o FoMO (por ejemplo, relacionados con el trabajo); (d) estudios de caso; (e) investigaciones instrumentales; ( $f$ ) trabajos que no realicen análisis de regresión.

Las investigaciones identificadas en cada uno de los recursos electrónicos fueron 86 en Web of Science, 19 en Scopus y 28 en PsycINFO, sumando un total de 133 trabajos que, tras aplicar los criterios establecidos, quedaron finalmente 11 investigaciones para revisión (Figura 1).

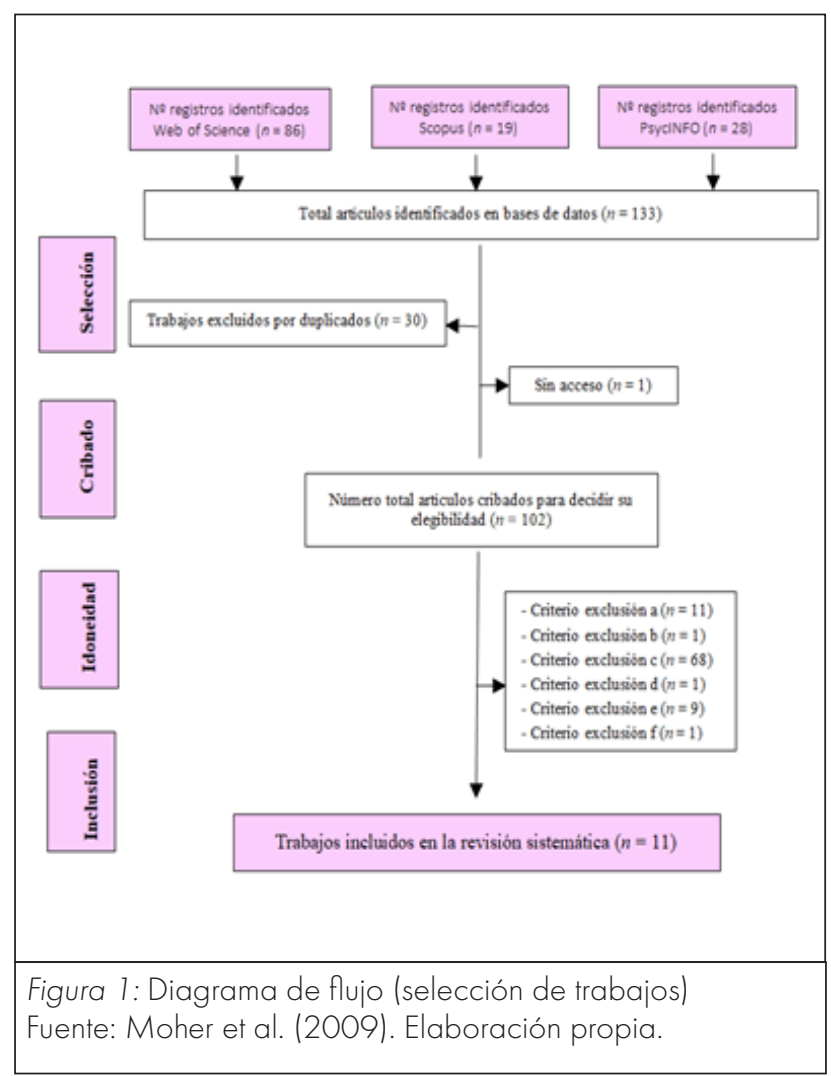




\section{RESULTADOS}

\section{ANÁLISIS DESCRIPTIVOS DE LOS TRABAJOS SELECCIONADOS}

La estrategia de búsqueda empleada y criterios establecidos arrojaron un total de 11 investigaciones para revisión, donde cada uno de los trabajos incluidos presentaba un objetivo diferente, sin embargo, tenían en común analizar la predicción del FoMO sobre distintas variables psicológicas.

Respecto al lugar donde se llevaron a cabo las investigaciones, dos de ellas se realizaron en el continente europeo, concretamente en Estonia (Gugushvili et al., 2020) y Alemania (Reer et al., 2019) y, otra en el continente asiático (Tang et al., 2020). Sin embargo, la mayoría se llevaron a cabo en América del Norte (Adams et a., 2020; Baker et al., 2016;
Barry et al., 2017, 2019; Elhai et al., 2016, 2020; Rogers et al., 2019; Scalzo y Martinez, 2017).

En relación a la población de estudio, la mayoría de los trabajos optaron por evaluar a adolescentes y sus padres, dada la influencia que ejerce el uso abusivo de las TIC sobre la personalidad (Barry et al., 2017, 2019).

Otras investigaciones prefirieron evaluar a estudiantes universitarios de diferentes edades (Adams et al., 2020; Baker et al., 2016; Elhai et al., 2020; Rogers et al., 2019; Scalzo y Martinez, 2017) o, incluso, a población en general (Elhai et al., 2016; Gugushvili et al., 2020; Reer et al., 2020; Tang et al., 2020).

En cuanto a las variables evaluadas en los diferentes trabajos, se pueden observar en la siguiente tabla 1 .

\section{Tabla 1 \\ Visión general de los trabajos incluidos en la revisión sistemática}

\begin{tabular}{|c|c|c|c|c|}
\hline $\begin{array}{c}\text { Autor y año de } \\
\text { publicación }\end{array}$ & Objetivo & Lugar & Población & Variables evaluadas \\
\hline $\begin{array}{l}\text { Adams et al. } \\
\text { (2020) }\end{array}$ & $\begin{array}{l}\text { Examinar las asociaciones de } \\
\text { estrés y FoMO con salud mental en } \\
\text { estudiantes universitarios y explorar si } \\
\text { estas relaciones eran por problemas } \\
\text { de sueño }\end{array}$ & Estados Unidos & $\begin{array}{l}\text { Estudiantes } \\
\text { universitarios }\end{array}$ & $\begin{array}{l}\text { Salud mental, insomnio y } \\
\text { estrés interpersonal }\end{array}$ \\
\hline Baker et al. (2016) & $\begin{array}{c}\text { Determinar si se asocia FoMO con } \\
\text { síntomas depresivos, atención plena } \\
\text { y síntomas físicos }\end{array}$ & Estados Unidos & $\begin{array}{l}\text { Estudiantes } \\
\text { universitarios }\end{array}$ & $\begin{array}{l}\text { FoMO, tiempo dedicado a } \\
\text { las redes sociales, síntomas } \\
\text { físicos, síntomas depresivos y } \\
\text { conciencia plena }\end{array}$ \\
\hline Barry et al. (2017) & $\begin{array}{c}\text { Investigar informes de adolescentes y } \\
\text { padres sobre el uso de redes sociales } \\
\text { y su relación con el ajuste psicosocial } \\
\text { adolescente }\end{array}$ & Estados Unidos & $\begin{array}{l}\text { Padres e hijos } \\
\text { adolescentes }\end{array}$ & $\begin{array}{l}\text { Uso redes sociales, lista de } \\
\text { verificación DMS (déficit de } \\
\text { atención/hiperactividad, } \\
\text { trastorno de conducta, } \\
\text { ansiedad y depresión), } \\
\text { FoMO y soledad }\end{array}$ \\
\hline Barry et al. (2019) & $\begin{array}{l}\text { Investigar la relación entre agresiones } \\
\text { y victimización a partir de los informes } \\
\text { de padres y adolescentes frente } \\
\text { al funcionamiento psicosocial y } \\
\text { participación en redes sociales }\end{array}$ & Estados Unidos & $\begin{array}{l}\text { Padres e hijos } \\
\text { adolescentes }\end{array}$ & $\begin{array}{l}\text { Participación en redes } \\
\text { sociales, narcisismo, } \\
\text { autoestima, FoMO y lista } \\
\text { de verificación DMS (déficit } \\
\text { de atención/hiperactividad, } \\
\text { trastorno de conducta, } \\
\text { ansiedad y depresión) }\end{array}$ \\
\hline Elhai et al. (2016) & $\begin{array}{l}\text { Examinar la relación entre el } \\
\text { uso problemático de teléfonos } \\
\text { inteligentes, depresión y ansiedad y, } \\
\text { posibles mecanismos de activación } \\
\text { conductual, necesidad de contacto, } \\
\text { FoMO y regulación emocional }\end{array}$ & Estados Unidos & $\begin{array}{l}\text { Población en } \\
\text { general }\end{array}$ & $\begin{array}{l}\text { Uso problemático de } \\
\text { teléfonos inteligentes, } \\
\text { FoMO, depresión, ansiedad, } \\
\text { activación conductual y } \\
\text { regulación emocional }\end{array}$ \\
\hline
\end{tabular}




\section{Tabla 1 (Continuación)}

Visión general de los trabajos incluidos en la revisión sistemática

\begin{tabular}{|c|c|c|c|c|}
\hline $\begin{array}{l}\text { Autor y año de } \\
\text { publicación }\end{array}$ & Objetivo & Lugar & Población & Variables evaluadas \\
\hline Elhai et al. (2020) & $\begin{array}{l}\text { Examinar si los síntomas de la } \\
\text { psicopatología (depresión, ansiedad } \\
\text { y FoMO) se asocian con el uso } \\
\text { de teléfonos inteligentes en redes } \\
\text { sociales y si se relaciona con el uso } \\
\text { problemático }\end{array}$ & Estados Unidos & $\begin{array}{l}\text { Estudiantes } \\
\text { universitarios }\end{array}$ & $\begin{array}{l}\text { Ansiedad, depresión, FoMO, } \\
\text { proceso y uso de teléfonos } \\
\text { inteligentes y adicción a } \\
\text { teléfonos inteligentes }\end{array}$ \\
\hline $\begin{array}{l}\text { Gugushvili et al. } \\
\text { (2020) }\end{array}$ & $\begin{array}{l}\text { Abordar el papel mediador del } \\
\text { uso problemático de Internet en la } \\
\text { asociación entre FoMO y bienestar } \\
\text { emocional }\end{array}$ & Estonia & $\begin{array}{l}\text { Población en } \\
\text { general }\end{array}$ & $\begin{array}{c}\text { Adicción al uso de teléfonos } \\
\text { inteligentes, FoMO y } \\
\text { bienestar emocional }\end{array}$ \\
\hline Reer et al. (2019) & $\begin{array}{l}\text { Investigar si la orientación de } \\
\text { comparación social y FoMO son } \\
\text { mediadores adicionales de la } \\
\text { conexión entre bienestar psicosocial } \\
\text { y compromiso a las redes sociales }\end{array}$ & Alemania & $\begin{array}{l}\text { Población en } \\
\text { general }\end{array}$ & $\begin{array}{l}\text { Ansiedad, depresión, } \\
\text { soledad, FoMO, orientación } \\
\text { de comparación social, } \\
\text { bienestar psicosocial y } \\
\text { compromiso a las redes } \\
\text { sociales }\end{array}$ \\
\hline $\begin{array}{l}\text { Rogers } \mathrm{e}^{\circ} \text { al } \\
\text { (2019) }\end{array}$ & $\begin{array}{l}\text { Probar los posibles efectos de } \\
\text { amortiguación de una intervención } \\
\text { educativa sobre el sueño y la } \\
\text { tecnología sobre las relaciones entre } \\
\text { FoMO y telepresión y sueño, uso de } \\
\text { redes sociales y uso de tecnología } \\
\text { disruptiva del sueño (es decir, uso de } \\
\text { tecnología antes y durante el sueño) }\end{array}$ & Estados Unidos & $\begin{array}{l}\text { Estudiantes } \\
\text { universitarios }\end{array}$ & $\begin{array}{l}\text { FoMO, telepresión general, } \\
\text { compromiso tecnológico } \\
\text { antes y durante el sueño, } \\
\text { uso de redes sociales y mal } \\
\text { hábito del sueño }\end{array}$ \\
\hline $\begin{array}{c}\text { Scalzo y Martinez } \\
\text { (2017) }\end{array}$ & $\begin{array}{l}\text { Examinar la relación entre uso de } \\
\text { bebidas alcohólicas, ansiedad y } \\
\text { FOMO }\end{array}$ & Estados Unidos & $\begin{array}{l}\text { Estudiantes } \\
\text { universitarios }\end{array}$ & $\begin{array}{c}\text { Ansiedad, intención de } \\
\text { beber y FoMO }\end{array}$ \\
\hline Tang et al. (2020) & $\begin{array}{l}\text { Examinar el papel mediador de } \\
\text { la tendencia a la adicción a } \\
\text { Internet, el miedo a perderse algún } \\
\text { acontecimiento (FOMO) y bienestar } \\
\text { psicológico en la relación entre la } \\
\text { exposición online a información } \\
\text { relacionada con el movimiento y } \\
\text { apoyo para acciones radicales }\end{array}$ & China & $\begin{array}{l}\text { Población en } \\
\text { general }\end{array}$ & $\begin{array}{l}\text { Exposición a la información } \\
\text { en línea, adicción a internet, } \\
\text { FoMO, aislamiento social, } \\
\text { depresión y apoyo a } \\
\text { acciones radicales }\end{array}$ \\
\hline $\begin{array}{c}\text { Scalzo y Martinez } \\
\text { (2017) }\end{array}$ & $\begin{array}{l}\text { Examinar la relación entre uso de } \\
\text { bebidas alcohólicas, ansiedad y } \\
\text { FoMO }\end{array}$ & Estados Unidos & $\begin{array}{l}\text { Estudiantes } \\
\text { universitarios }\end{array}$ & $\begin{array}{c}\text { Ansiedad, intención de } \\
\text { beber y FoMO }\end{array}$ \\
\hline
\end{tabular}

Por otro lado, el número de participantes en los diferentes trabajos varió de $N=97$ (Rogers et al., 2019) a $N=1929$ (Reer et al., 2019). Respecto a la edad, la más baja estuvo comprendida en el rango de 14-17 años (Barry et al., 2017, 2019) hasta los 1864 años (Baker et al., 2016).
La mayoría de las investigaciones para medir el nivel de FoMO utilizaron la escala de Przybylski et al. (2013), formada por 10 ítems. En este sentido, únicamente ha sido Gugushvili et al. (2020) quien optó por usar otro instrumento, concretamente, la escala de Raudlam (2018), tal y como se puede observar en lal siguiente Tabla 2: 


\begin{tabular}{|c|c|c|c|}
\hline \multicolumn{4}{|c|}{$\begin{array}{l}\text { Tabla } 2 \\
\text { Número de participantes, edad/media edad e instrumento evaluación FoMO }\end{array}$} \\
\hline $\begin{array}{l}\text { Autor y año de } \\
\text { publicación }\end{array}$ & $N$ & Edad/Media edad & $\begin{array}{l}\text { Instrumento evaluación } \\
\text { FOMO }\end{array}$ \\
\hline Adams et al. (2020) & 283 & $\begin{array}{l}18 \text { a } 50 \text { años (Edad Media } \\
21.40)\end{array}$ & $\begin{array}{l}\text { Escala FoMO de Przybylski et } \\
\text { al. (2013) }\end{array}$ \\
\hline Barry et al. (2019) & 428 & $\begin{array}{l}\text { Adolescentes con edad de } 14 \\
\qquad \text { a } 17 \text { años }\end{array}$ & $\begin{array}{l}\text { Escala FoMO de Przybylski et } \\
\qquad \text { al. (2013) }\end{array}$ \\
\hline Elhai et al. (2016) & 308 & $(M=33.15 \pm 10.21)$ & $\begin{array}{l}\text { Escala FoMO de Przybylski et } \\
\text { al. (2013) }\end{array}$ \\
\hline Elhai et al. (2020) & 316 & $\begin{array}{c}18 \text { a } 25 \text { años }(M=19.21 \pm \\
1.74)\end{array}$ & $\begin{array}{l}\text { Escala FoMO de Przybylski et } \\
\text { al. (2013) }\end{array}$ \\
\hline Gugushvili et al. (2020) & 426 & $\begin{array}{c}18 \text { a } 56 \text { años }(M=26.74 \pm \\
8.16)\end{array}$ & $\begin{array}{l}\text { La medida de FoMO de } \\
\text { Raudlam (2018) }\end{array}$ \\
\hline Reer et al. (2019) & 1929 & 14 a 39 años & $\begin{array}{l}\text { Escala FoMO de Przybylski et } \\
\text { al. (2013) }\end{array}$ \\
\hline Rogers et al. (2019) & 97 & $(M=19.81 \pm 2.55)$ & $\begin{array}{l}\text { Escala FoMO de Przybylski et } \\
\text { al. (2013) }\end{array}$ \\
\hline Scalzo y Martinez (2017) & 112 & $(M=18.91 \pm 1.05)$ & $\begin{array}{l}\text { Escala FoMO de Przybylski et } \\
\text { al. (2013) }\end{array}$ \\
\hline Tang et al. (2020) & 290 & (18 a 20 años) & $\begin{array}{l}\text { Escala FoMO de Przybylski et } \\
\text { al. (2013) }\end{array}$ \\
\hline te: Elaboración propia & & & \\
\hline
\end{tabular}

\section{FACTORES RELACIONADOS CON EL FOMO}

Entre los distintos factores psicológicos, como pueden ser ansiedad y/o depresión, Adams et al. (2020) hallaron como factores predictores del FoMO el estrés interpersonal, el insomnio y la salud mental. No obstante, no encontraronasociación positiva entre estas variables. En otra dirección, el FoMO sí se vio relacionado con el tiempo dedicado al uso de RRSS, diminución de salud física y psíquica (Baker et al., 2016) e incremento de niveles de ansiedad y síntomas depresivos (Barry et al., 2017).
Aquellos jóvenes que muestran mayores niveles de FoMO parecen poseer más probabilidades de ser víctimas de agresión o victimización a través de las RRSS (Barry et al., 2019).De hecho, se ha visto asociado que los adolescentes que presentaron un uso permanente de teléfonos inteligentes mostraban mayores síntomas ansiosos, depresivos (Elhai et al., 2020), FoMO y necesidad de coger el móvil (Elhai et al., 2016).

A su vez, Gugushvili et al. (2020) hallaron que el uso problemático de Internet mediaba parcialmente en la aparición del síndrome 
(Rogers et al., 2019). Además, los resultados de los trabajos que integran la presente revisión sistemática revelaron que un uso excesivo de redes sociales se encontró relacionado con un menor nivel de bienestar emocional, síntomas depresivos y ansiosos, y sentimientos de soledad (Reer et al., 2019). En otra dirección, entre las consecuencias de presentar un mayor nivel de ansiedad y FoMO en adolescentes, se ha visto relacionado las intenciones para realizar un consumo excesivo de bebidas alcohólicas (Tang et al., 2020).

\section{DISCUSIÓN}

Los resultados hallados en las distintas investigaciones nos han permitido alcanzar el objetivo del presente trabajo, que consistía en identificar mediante una revisión sistemática los factores psicológicos asociados al síndrome "miedo a perderse algún acontecimiento" (FoMO). Por su parte, Elhai et al. (2020), han relevado que el FoMO puede ser una respuesta dada a causa de una falta de afectividad, lo que conduce a un uso excesivo de Internet/teléfonos inteligentes para aliviar las emociones negativas. Desde esta perspectiva se podría explicar el por qué los universitarios que experimentan FoMO tratan de reducir su ansiedad o afecto negativo, puesto que tratan de buscar apoyo social a través del uso de las TIC y así poder regular sus emociones (Adams et al., 2020). Sin embargo, ello sugiere desfavorecer hacia un correcto ajuste emocional (Ferreira et al., 2021).

Otras líneas de investigación han señalado el impacto negativo que ejercen las relaciones orientadas al ciberespacio, es decir, preferencia por la amistad online sobre la amistad real. Sin embargo, el uso de las interacciones cara a cara con familiares y amigos son consideradas predictores del bienestar subjetivo, donde la falta de dichas interacciones puede explicar el impacto negativo que presenta el FoMO en el bienestar emocional al verse asociada positivamente con sentimientos de soledad en adolescentes y adultos (Reer et al., 2019), por lo que se ha podido alcanzar el primer objetivo específico planteado en el presente trabajo.

Por otro lado, los resultados también han permito logar el segundo objetivo específico de la investigación. En este sentido, los adolescentes que muestran niveles más elevados en el síndrome, sugieren que este predice variables como la victimización, intención de un consumo excesivo de bebidas alcohólicas y afectación en la calidad del sueño. Particularmente, las experiencias de agresión y victimización se han visto asociadas con una mayor frecuencia del uso de las RRSS y número de cuentas virtuales (Facebook, WhatsApp, Instagram...), relacionas con la falta de atención por parte de los padres y el miedo a perderse algún acontecimiento (Barry et al., 2017). A su vez, los adolescentes que ejercen violencia "agresores" presentan dificultades emocionales y son personas narcisistas, mientras que las víctimas, en la mayoría de los casos muestran síntomas depresivos (Elhai et al., 2020), baja autoestima y soledad (Reer et al., 2019), lo que les hace más vulnerables a este hecho.

Por tanto, sería interesante que los padres estuviesen informados en la forma en que utilizan sus hijos los dispositivos inteligentes durante la etapa adolescente, involucrándose en sus experiencias online, de modo que les garanticen hacia un desarrollo integral (Barry et al., 2019). Estos resultados están en línea con los expuestos por Montes-Vozmediano et al. (2020), donde la vigilancia de los padres sugiere ser un factor protector sobre la aparición del síndrome en jóvenes (Martínez, 2021).

En el presente trabajo se ha podido observar que mayores niveles de FoMO se encuentran vinculados a un uso excesivo de RRSS (Gugushvili et al., 2020; Rogers y Barber, 2019). De este modo, la presencia de este síndrome podría derivar en problemas relacionados con la calidad del sueño, tristeza e insatisfacción con la vida en población adolescente (Riordan et al., 2015). Así 
como, con un incremento de estrés, lo que conduce a una mayor intención para realizar un consumo excesivo de bebidas alcohólicas (Scalzo y Martinez, 2017; Tang et al., 2020).

En definitiva, el uso excesivo de RRSS, tanto en población adolescente como adulta influye de manera negativa el bienestar de la persona, ya que favorece a que se creen interacciones enriquecedoras cara a cara con familiares y/o amigos. En particular, habría que tener en cuenta que las relaciones socioafectivas son relevantes en la etapa de la adolescencia por su impacto sobre el correcto ajuste psicológico y desarrollo de la personalidad.

Los resultados obtenidos tienen una serie de implicaciones a nivel teórico y práctico. En primer lugar, respecto a las implicaciones teóricas, el presente trabajo es la primera investigación que se ha enfocado explícitamente en investigar y presentar predictores psicológicos asociados al Síndrome FoMO, abarcando población adolescente y adulta. Por otro lado, respecto a las implicaciones prácticas, conocer factores de riesgo de padecer Síndrome de FoMO es el paso previo necesario para diseñar programas preventivos eficaces. s ser tilizada No obstante, este trabajo no se encuentra exento de limitaciones. La principal limitación podría presentarse en los recursos electrónicos utilizados para la búsqueda de trabajos. Puesto que, en nuestro caso, hemos hecho uso de las bases de datos Web of Science, Scopus y PsycINFO, por lo que es posible que se hayan podido omitir involuntariamente trabajos publicados en otros recursos electrónicos. En segundo lugar, se ha evidenciado una falta de estudios de diseño longitudinal. Este hecho ha impedido que no se pudiese realizar un metaanálisis para comprobar la eficacia que poseen los programas de intervención sobre el FoMO. Por último, solamente se han analizado trabajos de regresión, con lo que las investigaciones basadas en análisis de diferencias, correlaciones $u$ otro tipo de relaciones no se han incluido en el presente estudio.
Para futuras líneas de investigación sería interesante que nuevos trabajos incorporasen en sus investigaciones nuevas variables como, por ejemplo, la inteligencia emocional, dada la influencia que presenta en el sujeto sobre e uso correcto o incorrecto de dispositivos inteligentes (Martínez, 2021). De esta manera se podrían establecer nuevas conclusiones.

En resumen, el síndrome de FoMO pareceencuentrarse relacionado con un mayor uso de RRSSo teléfonos inteligentes. Las personas que prefieren tener relaciones basadas en la red presentan una peor salud psicológica y física. Particularmente, muestran mayores niveles en ansiedad, depresión, sentimientos de soledad, intenciones conductuales para realizar un consumo excesivo de alcohol, un menor nivel de bienestar emocional, un incremento de conductas de agresión o victimización, así como una afectación en lo que respecta a la calidad del sueño.

\section{- Conflicto de intereses}

Los autores declaran no tener ningún conflicto de interés.

\section{REFERENCIAS}

*Adams, S.K., Murdock, K.K., Daly-Cano, M., y Rose, M. (2020). Sleep in the socialworld of college students: Bridging interpersonal stress and fear of missing out with mental health. Behavioral Sciences, 10(2), 54. https://doi.org/10.3390/bs 10020054

*Baker, Z.G., Krieger, H., y LeRoy, A.S. (2016). Fear of missing out: Relationships with depression, mindfulness, and physical symptoms. Translational Issues in Psychological Science, 2(3), 275-282. https://doi.org/10.1037/tps0000075

*Barry, C.T., Sidoti, C.L., Briggs, S.M., Reiter, S.R., y Lindsey, R.A. (2017). Adolescent social media use and mental health from adolescent and parent perspectives. Journal of Adolescence, 61, 1-11. https://doi.org/10.1016/i. adolescence.2017.08.005

* Barry, C.T., Briggs, S.M., y Sidoti, C. (2019). Adolescent and Parent Reports of Aggression and Victimization on Social Media: Associations With Psychosocial 
Adjustmen. Journal of Child and Family Studies, 28, 2286-2296. https://doi. org/10.1007/s 10826-019-01445-1

Brito, D., Yasmin, J., González, V.A., Preciado, M., y Abreus, J.L. (2021). Influencia de la utilización de las redes sociales en el proceso de comunicación interpersonal. Revista Científica, Cultura, Comunicación y Desarrollo, 6(3), 6-13. https://rccd.ucf. edu.cu/index.php/aes/issue/view/15

Budnick, C.J., Rogers, A.P., y Barber, L.K. (2020). The fear of missing out at work: Examining costs and benefits to employee health and motivation. Computers in Human Behavior, 104, 1-13. https://doi. org/10.1016/i.chb.2019.106161

Camacho, E., LoPresti, M., Appelboom, G., Dumont, E.L.P., Taylor, B., y Connoly, E.S. (2014). The ubiquitous role of smartphones in mobile health. Biometrics \& Biostatistics International Journal, 1(1), 14-19. https:// doi.org/10.15406/bbii.2014.01.00004

Chan, M. (2015). Mobile phones and the good life: Examining the relationships among mobile use, social capital and subjective well-being. New Media \& Society, 17(1), 96-113. https://doi. org/10.1177/1461444813516836

Coiro, M.J., Bettis, A.H., y Compas, B.E. (2017). College students coping with interpersonal stress: Examining a controlbased model of coping. Journal of American College Health, 65(3), 177 186. https://doi.org/10.1080/0744848 1.2016 .1266641

Dhir, A., Yossatorn, Y., Kaur, P., y Chen, S. (2018). Online social media fatigue and psychological wellbeing-A study of compulsive use, fear of missing out, fatigue, anxiety and depression. International Journal of Information Management, 40, 141-152. https://doi.org/10.1016/i. iiinfomgt.2018.01.012

Duke, É., y Montag, C. (2017). Smartphone addiction, daily interruptions and self-reported productivity. Addictive Behaviors Reports, 6, 90-95. https://doi. org/10.1016/i.abrep.2017.07.002

Elhai, J.D., Gallinari, E.F., Rozgonjuk, D., y Yang, H. (2020). Depression, anxiety and fear of missing out as correlates of social, non-social and problematic smartphone use. Addictive Behaviors, 105, 1-7. https://doi.org/10.1016/i. addbeh.2020.106335

* Elhai, J.D., Levine, J.C., Dvorak, R.D., y Hall, B.J. (2016). Fear of missing out, need for touch, anxiety and depression are related to problematic smartphone use. Computers in Human Behavior, 63, 509-516. $\quad$ https://doi.org/10.1016/i. chb.2016.05.079

Elsevier (2019). Mendeley. Elsevier. https:// www.mendeley.com/download-desktop.

Ferreira, D., De Sousa, H., De Moraes, G., De Assis, S.E., Negreiros, F., y Medeiros, E.D. (2021). Fear of missing out (FoMO), mídias sociais e ansiedade: Uma revisão sistemática. Psicología, Conocimiento y Sociedad, 17 (3), 99-114. https://dx.doi. org/10.26864/pcs.v11.n3.7

Godwin-Jones, R. (2011). Emerging technologies: Mobile apps for language learning. Language Learning \& Technology, 15(2), 2-11. http://dx.doi. org/10125/44244

*Gugushvili, N., Täht, K., Rozgonjuk, D., Raudlam, M., Ruiter, R., y Verduyn, P. (2020). Two dimensions of problematic smartphone use mediate the relationship between fear of missing out and emotional well-being. Cyberpsychology, 14(2), 1-22. https://doi.org/10.5817/CP2020-2-3

Lo Coco, G., Salerno, L., Franchina, V., La Tona, A., Di Blasi, M., y Giordano, C. (2020). Examining bi-directionality between Fear of Missing Out and problematic smartphone use. A twowave panel study among adolescents. Addictive Behaviors, 106, 1-6. https://doi. org/10.1016/i.addbeh.2020.106360

Martínez, M. (2021). Relación existente entre el uso problemático del teléfono móvil e internet y adolescencia. OIDLES. Desarrollo Local y Economía Social, [In press], 57-70. https://www.eumed.net/es/ revistas/oidles/especial-noviembre-21/ internet-adolescencia

Moher, D., Liberati, A., Tetzlaff, J., Altman, D.G., Altman, D., Antes, G., Atkins, D., Barbour, V., Barrowman, N., Berlin, J.A., Clark, J., Clarke, M., Cook, D., D'Amico, R., Deeks, J.J., Devereaux, P.J., Dickersin, K., Egger, M., Ernst, E...Tugwell, P. 
(2009). Preferred reporting items for systematic reviews and meta-analyses: The PRISMA statement. Annals of Internal Medicine, 151(4), 264-269. https:// doi.org/10.7326/0003-4819-151-4200908180-00135

Montes-Vozmediano, M., Pastor, Y., Martín-Nieto, R., y Atuesta, J.D. (2020). Smartphone y redes sociales: una aproximación a los usos, vulnerabilidades y riesgos durante la adolescencia en España y Colombia. Revista Espacios, 41 (4), 44-59. https://doi.org/10.48082/ espacios-a20v4 1n48p04

Primack, B.A., Shensa, A., Sidani, J.E., Whaite, E.O., Lin, L., Rosen, D., Colditz, J.B., Radovic, A., y Miller, E. (2017). Social Media Use and Perceived Social Isolation Among Young Adults in the U.S. American Journal of Preventive Medicine, 53(1), 1-8. https://doi.org/10.1016/i. amepre.2017.01.010

Przybylski, A.K., Murayama, K., DeHaan, C.R., y Gladwell, V. (2013). Motivational, emotional, and behavioral correlates of fear of missing out. Computers in Human Behavior, 29(4), 1841-1848. https://doi. org/10.1016/i.chb.2013.02.014

Raudlam, M. (2018). Ilmajäämishirmu (FoMO) küsimustiku adapteerimine ja valideerimine eesti keelde. Adaptation and validation of Estonian fear of missiong out (FoMO) scale. Unpublished Master's thesis. University of Tartu, Estonia.

*Reer, F., Tang, W.Y., y Quandt, T. (2019). Psychosocial well-being and social media engagement: The mediating roles of social comparison orientation and fear of missing out. New Media and Society, $21(7), 1486-1505$. https://doi. org/10.1177/1461444818823719

Riordan, B.C., Flett, J.A.M., Hunter, J.A., Scarf, D., y Conner, T.S. (2015). Fear of missing out (FoMO): the relationship between FoMO, alcohol use, and alcoholrelated consequences in college students. Journal of Psychiatry and Brain Functions, 2(1), 9. https://doi.org/10.7243/20553447-2-9
Robinson, A., Bonnette, A., Howard, K., Ceballos, N., Dailey, S., Lu, Y., y Grimes, T. (2019). Social comparisons, social media addiction, and social interaction: An examination of specific social media behaviors related to major depressive disorder in a millennial population. Journal of Applied Biobehavioral Research, 24(1), e12158. https://doi.org/10.1111/ jabr. 12158

*Rogers, A.P., y Barber, L.K. (2019). Computers in Human Behavior, 93, 192-199. https:// doi.org/10.1016/i.chb.2018.12.016

Rozgonjuk, D., Kattago, M., y Täht, K. (2018). Social media use in lectures mediates the relationship between procrastination and problematic smartphone use. Computers in Human Behavior, 89, 191-198. https:// doi.org/10.1016/i.chb.2018.08.003

Scalzo, A.C., y Martinez, J.A. (2017). Not all anxiety is the same: How different "types" of anxiety uniquely associate with college students' drinking intentions. Journal of College Student Development, 58(6), 943-947. https://doi.org/10.1353/ csd.2017.0073

Tang, G., Hung, E.P.W., Au-Yeung, H.K.C., y Yuen, S. (2020). Politically motivated internet addiction: Relationships among online information exposure, internet addiction, FOMO, Psychological wellbeing, and radicalism in massive political Turbulence. International Journal of Environmental Research and Public Health, 17(2), 633. https://doi.org/10.3390/ ijerph17020633 